\title{
Abnormal neutrophil-to-lymphocyte ratio in children with autism spectrum disorder and history of maternal immune activation
}

Pierre Ellul ( $\sim$ pierre.ellul@aphp.fr)

Robert Debre Hospital, APHP

\section{Anna Maruani}

Robert Debre Hospital, APHP

Hugo Peyre

Robert Debre Hospital, APHP

Valérie Vantalon

Robert Debre Hospital, APHP

Daphnée Hoareau

Robert Debre Hospital, APHP

Hugo Tiercelin

Robert Debre Hospital, APHP

Michelle Rosenzwajg

Sorbonne Université

David Klatzmann

Sorbonne Université

Richard Delorme

Robert Debre Hospital, APHP

\section{Research Article}

Keywords: Neurodevelopmental disorders, Immunology, Biomarkers, Biotherapy

Posted Date: September 21st, 2022

DOI: https://doi.org/10.21203/rs.3.rs-1334630/v2

License: (1) (i) This work is licensed under a Creative Commons Attribution 4.0 International License.

Read Full License 


\section{Abstract}

Background: Maternal immune activation (MIA), related to autoimmune/inflammatory diseases or acute infections, during the two first trimesters of pregnancy is a risk factor for autism spectrum disorders (ASD) in offspring. In mice, MIA has a long-term impact on offspring's immune equilibrium resulting in a pro-inflammatory phenotype.

Methods: We therefore hypothesized that children with ASD and a history of MIA could display a similar phenotype such as a higher neutrophil to lymphocyte ratio (NLR). In this study, we used a retrospective sample of 231 dyads involving children with ASD and their mothers.

Results: Among ASD patients, $12 \%$ had a history of MIA. The multivariate analysis revealed a significant association between NLR in children with ASD and maternal history of MIA ( $F=2.27, p=0.03)$. Using a categorical approach, we observed an abnormal NLR (over 3 ) in $7.4 \%$ of children with ASD MIA+ compared to $1.9 \%$ for MIA-

Conclusion: Our study reinforced preliminary evidence suggesting an impact of MIA on the risk of ASD. Further studies could contribute to the development of biomarkers in MIA+ ASD and enable the development of targeted immunomodulatory therapies.

\section{Background}

Autism spectrum disorders (ASD) are a heterogeneous group of neurodevelopmental disorders [1] with an estimated prevalence of approximately 1 in 100 [2]. ASD are characterized by a deficit in social communication associated with restricted and repetitive behaviors [3]. ASD result of a complex interplay between genetics, epigenetics and environmental factors [4].

Preclinical and epidemiological studies highlight the role of maternal immune activation (MIA) during pregnancy, whether due to autoimmune/inflammatory diseases or acute infections, as a risk factor for ASD in the offspring [5]. Mice models show the central role of maternal cytokines in the disruption of fetal brain development. The maternal secretion of interleukin 6 (IL6) and 17a (IL17a) during gestation plays a pivotal role in the development of ASD-like behaviors in pups [6, 7]. MIA exposure also induces longlasting changes in the offspring's immune system. In utero exposure to MIA leads to increased activation of Th17 lymphocytes - a pro-inflammatory subset secreting IL17a - and a decrease of the regulatory T lymphocytes (Tregs), in the offspring through potential epigenetic mechanisms [8, 9]. Similar deregulations were recently reported in children of women who were affected by the SARS-Cov2 during pregnancy [10].

The neutrophil/lymphocyte ratio (NLR) is an indicator of impaired cell-mediated immunity, frequently associated with inflammation [11] and could represent an immune stigma in the offspring in case of MIA during pregnancy. Higher NLR have been correlated to negative prognostic in several human diseases and is also associated with all-cause mortality in the general population $[12,13]$. In recent years, there is a 
growing interest in NLR in psychiatric disorders suspected to be related to an immune deregulation such as schizophrenia, bipolar disorders or depression [14]. To our knowledge, only two studies examined NLR in ASD, but with inconclusive results $[15,16]$. None of these reports however assessed the impact of MIA in the NLR variability. In the present study, we retrospectively explored the NLR in children with ASD and a history of active MIA during pregnancy. We hypothesized that offspring with ASD and MIA (MIA+) would display an increased NLR compared to those with ASD without MIA (MIA-).

\section{Methods}

\section{Participants}

We included in our study children with ASD who were part of the PARIS study, conducted by the Excellence Centre for Autism \& Neuro-developmental Disorders (InovAnd) at the Robert Debré Hospital between March 2017 to April 2021. This study was approved by the local ethics committee (2021-27 $\mathrm{N}^{\circ}$ IDRCB: 2021-A00489-32). Informed consents were obtained before enrollment in the study.

All participants were screened using a parental semi-structured interview for medical history. The final diagnosis of ASD was performed according to DSM-5 criteria [3] by summing up the information from the Autism Diagnostic Interview-Revised [17], the Autism Diagnostic Observation Schedule - 2nd edition (ADOS-2) [18] and clinical records of the individuals. Pre- and peri- natal history was evaluated through a direct semi-structured interview with the mother of each child enrolled in the study. We paid a specific attention to a history of MIA by scrutiny explore any diagnosed immune mediated illnesses which could have occurred during pregnancy. Based on this information, children were then split either in MIA (MIA+) or in non-MIA (MIA-) sub-groups. We considered mothers with a significant history of a MIA-related event during pregnancy when they were: (i) with an autoimmune disease as listed by the American Autoimmune Related Diseases Association: https://www.aarda.org/diseaselist. The disease should have occurred during the first or the second trimester of pregnancy, or was present before the pregnancy and had a flare-up requesting a treatment adjustment during pregnancy; (ii) with a viral or bacterial infection during pregnancy with a fever over $38.5^{\circ} \mathrm{C}$ for more than 24 hours. Occurring during the first or the second trimester of pregnancy. Mothers with an infection resulting from a pathogen with a well-documented direct brain cytopathic effect (such as cytomegalovirus infection) were excluded (iii) with gestational diabetes. We only considered mothers requiring insulin supplementation. We considered that this condition was more likely to be associated with a significant systemic metabolic inflammation [18].

Neutrophil to lymphocyte ratio

Routine blood counting was performed using XN 3000 (Sysmex) NLR was then calculated by dividing the absolute value of the neutrophil count by the absolute value of the lymphocyte count. In the categorical analysis, a NLR greater than 3 was considered as pathological [19].

\section{Statistical analysis}


Before each analysis, the normality of the distribution of the variables was tested with a Shapiro-Wilk test. Parametric or non-parametric tests were used accordingly. For continuous variables, we used independent sample t-tests, or Wilcoxon for continuous variables. For categorical variables, the Fisher test was used.

In linear models, only models with a normal distribution of residuals were used. Hierarchical regression analysis are in supplementary Table 2. The final model of multivariate analysis was thus adjusted on age, gender and pregnancy complications (placenta previa and maternal-fetal infections). Statistical analysis was performed using $\mathrm{R}$ studio version 4.2.1.

\section{Results}

\section{General characteristics of the offspring with ASD}

Among the 231 mother-child dyads included in the study, a MIA during pregnancy was found in $11.68 \%$ of mothers $(n=27)(M I A+)$ (Supplementary Table 1$)$. 
Table 1

Main characteristics of autism spectrum disorder patients with or without a history of maternal immune activation.

\begin{tabular}{|c|c|c|c|}
\hline & MIA+ & MIA- & $p$ value \\
\hline Numbers of patients & 27 & 204 & \\
\hline \multicolumn{4}{|l|}{ Offspring } \\
\hline Male/ Female, n (\%) & 22/5 (81/19) & $159 / 44(78 / 22)$ & 0.89 \\
\hline Age at inclusion [mean (SD)] & $78.22(24.84)$ & 89.55 (25.89) & $0.03^{*}$ \\
\hline \multicolumn{4}{|l|}{ Pregnancy complications, n (\%) } \\
\hline Consanguinity & $0(0)$ & $16(7.8)$ & 0.22 \\
\hline Medically-assisted procreation & $0(0)$ & $12(5.88)$ & 0.36 \\
\hline History of spontaneous miscarriage & $10(37.03)$ & $43(21.07)$ & 0.1 \\
\hline Folate supplementation & $16(59.25)$ & $86(42.15)$ & 0.14 \\
\hline Threat of premature delivery & $1(3.70)$ & $8(3.92)$ & 1 \\
\hline Arterial hypertension & $1(3.70)$ & $9(4.41)$ & 1 \\
\hline Placenta previa & $2(7.40)$ & $1(0.49)$ & $0.03^{\star}$ \\
\hline Premature rupture of membranes & $2(7.40)$ & $2(0.98)$ & 0.06 \\
\hline Intrauterine growth retardation & $1(3.70)$ & $7(3.43)$ & 1 \\
\hline Macrosomia & $0(0)$ & $2(0.98)$ & 1 \\
\hline Maternal-foetal infection & $3(11.1)$ & $3(1.47)$ & $0,02^{\star}$ \\
\hline \multicolumn{4}{|l|}{ Birth parameters, mean (SD) } \\
\hline Birth term & $38.53(1.39)$ & $38.58(2.23)$ & 0.06 \\
\hline Birth height & $48.84(2.34)$ & $49.46(3.54)$ & $0.04 *$ \\
\hline Birth weight & $3273(452.01)$ & $3289(658.24)$ & 0.47 \\
\hline Head circumference & $35.62(3.78)$ & $34.76(3.02)$ & 0.31 \\
\hline 1-minute Apgar & $9.44(1.08)$ & $9.44(1.49)$ & 0.43 \\
\hline 5-minute Apgar & $9.88(0.42)$ & $9.87(0.73)$ & 0.51 \\
\hline \multicolumn{4}{|c|}{ Biological parameters (univariate analysis) } \\
\hline Neutrophils & $3.30(1.89)$ & $3.15(1.35)$ & 0.90 \\
\hline
\end{tabular}

MIA: Maternal immune activation; SD: standard deviation; 


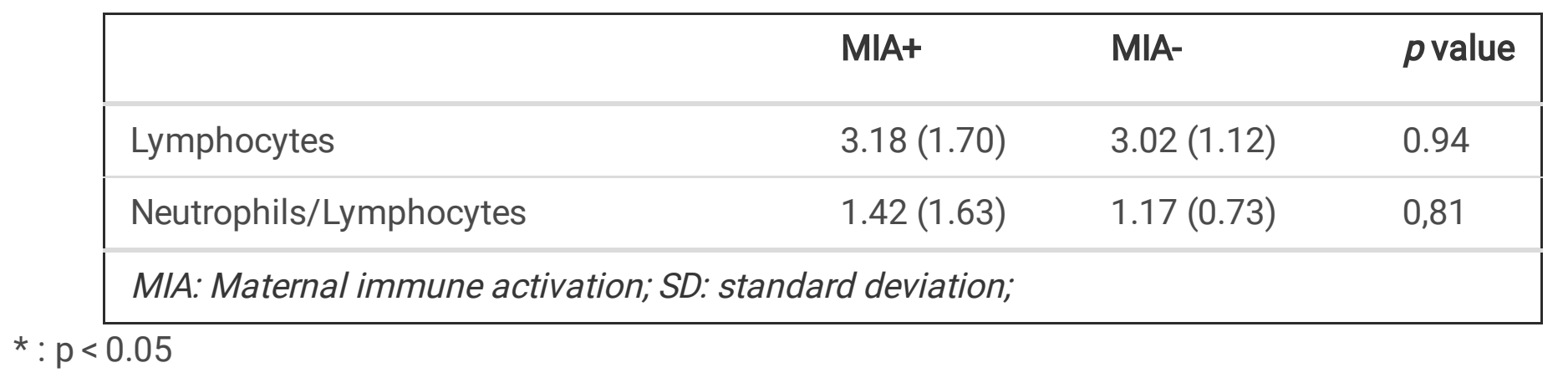

Dyads without MIA during pregnancy ( $n=204,88.31 \%$ ) were used as a comparison group (MIA-). Demographics characteristics of the population were provided in Table 1. The mean age of offspring with ASD was significantly lower in the MIA + group $(78.2+/-24.8$ vs $89.6+/-25.9$ months, $p=0.033)$. In accordance with literature, we observed that mothers from MIA + group have had more pregnancy complications than those in the MIA-group, with significant differences for placenta previa $(p=0.03)$ and maternofetal infection at birth $(p=0.02)$. Concerning offspring, we found that offspring from MIA + have a lower birth height $(p=0.04)$. We did not report any other significant difference concerning birth parameters and offspring's medical history.

\section{NLR variability}

We first explored the NLR variability by performing a univariate analysis. We observed no significant difference between the MIA + and MIA- sub-groups considering neutrophils $\left(3.30+/-1.89 \times 10^{\wedge} 9 / L\right.$ vs 3.15 $\left.+/-1.35 \times 10^{\wedge} 9 / L ; p=0.68\right)$, lymphocytes $\left(3.18+/-1.7 \times 10^{\wedge} 9 / L\right.$ vs $\left.3.02+/-1.12 \times 10^{\wedge} 9 / L ; p=0.63\right)$ or the $\operatorname{NLR}(1.42+/-1.63$ vs $1.17+/-0.73 ; p=0.43)$.

We then performed a multivariate analysis by incorporating potential confounding factors. (Table 2). 
Table 2

Details of multivariate linear regression analysis

\begin{tabular}{|c|c|c|c|}
\hline Lymphocytes & Beta & $\mathbf{t}$ & p-value \\
\hline \multicolumn{4}{|l|}{ Model 1} \\
\hline MIA & 0,04 & 0,65 & 0,51 \\
\hline \multicolumn{4}{|l|}{ Model 2} \\
\hline MIA & 0,009 & 0,14 & 0,89 \\
\hline Age & $-0,22$ & $-3,32$ & 0,001 \\
\hline Gender & 0,06 & 0,89 & 0,38 \\
\hline \multicolumn{4}{|l|}{ Model 3} \\
\hline MIA & 0,005 & 0,08 & 0,94 \\
\hline Age & $-0,21$ & $-3,22$ & 0,001 \\
\hline Gender & 0,05 & 0,75 & 0,45 \\
\hline MFI & $-0,06$ & $-0,95$ & 0,34 \\
\hline Placenta Previa & 0,09 & 1,29 & 0,2 \\
\hline Neutrophiles & Beta & $t$ & p-value \\
\hline \multicolumn{4}{|l|}{ Model 1} \\
\hline MIA & 0,034 & 0,52 & 0,6 \\
\hline \multicolumn{4}{|l|}{ Model 2} \\
\hline MIA & 0,06 & 0,89 & 0,37 \\
\hline Age & 0,16 & 2,47 & 0,01 \\
\hline Gender & 0,004 & 0,06 & 0,95 \\
\hline \multicolumn{4}{|l|}{ Model 3} \\
\hline MIA & 0,08 & 1,15 & 0,25 \\
\hline Age & 0,17 & 2,56 & 0,01 \\
\hline Gender & 0,003 & 0,043 & 0,97 \\
\hline MFI & $-0,05$ & $-0,77$ & 0,44 \\
\hline Placenta Previa & $-0,04$ & $-0,63$ & 0,53 \\
\hline NLR & Beta & $t$ & p-value \\
\hline
\end{tabular}




\begin{tabular}{|lccl|}
\hline Lymphocytes & Beta & $\mathbf{t}$ & p-value \\
\hline Model 1 & & & \\
MIA & 0,09 & 1,38 & 0,17 \\
Model 2 & & & \\
MIA & 0,12 & 1,91 & 0,06 \\
Age & 0,21 & 3,19 & 0,002 \\
Gender & $-0,08$ & $-1,31$ & 0,19 \\
Model 3 & & & \\
\hline MIA & 0,14 & 2,14 & $0,03^{\star}$ \\
\hline Age & 0,21 & 3,21 & 0,001 \\
\hline Gender & $-0,08$ & $-1,26$ & 0,21 \\
\hline MFI & $-0,02$ & $-0,33$ & 0,74 \\
\hline Placenta Previa & $-0,08$ & $-1,15$ & 0,25 \\
\hline
\end{tabular}

MIA: Maternal immune activation; MFI: Materno-fetal infection

$\star: p<0.05$

We found a significant increase of the NLR in the MIA + offspring $(F=3.04, p=0.03)$ but not with the concentrations of lymphocytes $(p=0.94)$ and neutrophils $(p=0.25)$.

Using a categorical approach, there was a tendency for more frequent pathological value of NLR (NLR over 3$)$ in the MIA + than in the MIA-subgroups [7.4\% (2/27) vs $1.9 \%(4 / 204), p=0.14]$.

\section{Discussion}

In accordance with our initial hypothesis, we reported that NLR was significantly higher in ASD children with a history of MIA than in ASD without MIA. Our results fostered preliminary evidence suggesting that ASD patients with a history of MIA have persistent peripheral inflammation and that NLR may be a potential biomarker of this immune dysregulation.

ASD by itself are associated with an immune dysregulation such as a decrease in Tregs and an increase in Th17 [20]. In animal models of ASD, MIA also induced long term increase of the Th17 [8] and decrease of Tregs in offspring [9]. This Tregs/Th17 imbalance results in a polarization of the immune balance toward a peripheral inflammation. Although we lack a control group of children without ASD born to mothers with MIA to independently assess MIA impact on the NLR of the offspring, recent basic neuroimmunology studies emphasized the importance of IL-17a in brain development but also in the homeostasis of cognitive function [21,22]. Also, Tregs have been recently discovered in the perineuronal 
net and could play a fundamental role in neuronal homeostasis [23]. Moreover, in a mouse model of MIAinduced ASD, Tregs transplantation leads to normalization of autistic behaviors [24]. These data highlight that the immune dysregulation induced by MIA could have a pathophysiological impact in ASD children.

Using NLR, our study was the first to confirm preclinical findings in autistic individuals with a history of MIA. To our knowledge, only two previous studies have explored NLR in ASD. They reported no association with ASD but without considering the heterogeneity of individuals regarding MIA $[15,16]$. The authors however showed a trend for a significant correlation between NLR and autistic symptom severity. Interestingly, MIA during pregnancy was also associated with severe ASD related features in the offspring [25]. Taken together with the literature, our results highlighted the potential role of immunity deregulation, and specifically the Tregs/Th17 imbalance, in the pathophysiology of ASD.

In our sample, we also observed more pregnancy complications in the MIA + group. Numerous examples in the literature emphasize that neurodevelopmental disorders may emerge through the addition of causal factors [5]. In animal studies, MIA is considered as a disease primer, making the offspring more susceptible to a second hit that might precipitate the rise of neurodevelopmental disorders [26]. The overrepresentation of perinatal hitches in the MIA + individuals may indirectly reflect this additive model of determinism in ASD [29]. Although no studies have investigated this hypothesis in the context of MIA, future cohort studies may try to further decipher the intrinsic link between MIA in pregnant mothers and the development of ASD in offspring. We advocate that they adopt a longitudinal perspective and take into account (i) 'secondary' events, (ii) clinical phenotyping of patients (severity of autism co-morbidity, ADHD co-morbidity) and (iii) include a deep immunophenotyping approach on larger samples. Our study has to be considered in light of its limitations. First, due to the intrinsic nature of our study, the collection of data and in particular events during pregnancy was retrospective and may therefore be subject to recall bias. Secondly, our sample size was small, particularly for the MIA + group. These two limitations were balanced by the use of stringent criteria on the definition of active MIA during pregnancy allowing (i) to temper recall bias and (ii) to define a homogeneous and powerful MIA + sample, enough to detect subtle differences between groups., in the general population infection during pregnancy is very frequent [28]. Thus, trying to make association between this common events with a less common occurrence (ASD in the offspring) carries the risk of false associations. It is important to note, however, that our results are consistent with those found in basic research studies. Fifth, the NLR cut-off used in categorical analysis is only validated in an adult population. To our knowledge, no such threshold exists for the pediatric population [29].

\section{Conclusions}

In ASD, we showed that history of MIA seems to be associated with long term peripheral immune deregulation. Further studies are needed to define how this peripheral inflammation contributes to the pathophysiology of autism. Indeed, there is still a lack of precise data on the interaction between peripheral inflammation and brain function in autism. However, we can hope that the translation of 
knowledge gained from animal models to patients, using deep immunophenotyping data, will allow us to fill gaps in our knowledge and open new avenues in the development of immunotherapy in ASD.

\section{References}

1. Lord C, Brugha TS, Charman T, et al (2020) Autism spectrum disorder. Nat Rev Dis Primer 6:5. https://doi.org/10.1038/s41572-019-0138-4

2. Maenner MJ, Shaw KA, Baio J, et al (2020) Prevalence of Autism Spectrum Disorder Among Children Aged 8 Years - Autism and Developmental Disabilities Monitoring Network, 11 Sites, United States, 2016. Morb Mortal Wkly Rep Surveill Summ Wash DC 2002 69:1-12. https://doi.org/10.15585/mmwr.ss6904a1

3. (2013) Diagnostic and statistical manual of mental disorders: DSM-5, 5th ed. American psychiatric association, Washington

4. Schaaf CP, Betancur C, Yuen RKC, et al (2020) A framework for an evidence-based gene list relevant to autism spectrum disorder. Nat Rev Genet 21:367-376. https://doi.org/10.1038/s41576-020-02312

5. Han VX, Patel S, Jones HF, Dale RC (2021) Maternal immune activation and neuroinflammation in human neurodevelopmental disorders. Nat Rev Neurol 17:564-579. https://doi.org/10.1038/s41582021-00530-8

6. Choi GB, Yim YS, Wong H, et al (2016) The maternal interleukin-17a pathway in mice promotes autism-like phenotypes in offspring. Science 351:933-939.

https://doi.org/10.1126/science.aad0314

7. Smith SEP, Li J, Garbett K, et al (2007) Maternal immune activation alters fetal brain development through interleukin-6. J Neurosci Off J Soc Neurosci 27:10695-10702. https://doi.org/10.1523/JNEUROSCI.2178-07.2007

8. Lim Al, McFadden T, Link VM, et al (2021) Prenatal maternal infection promotes tissue-specific immunity and inflammation in offspring. Science 373:eabf3002. https://doi.org/10.1126/science.abf3002

9. Hsiao EY, McBride SW, Chow J, et al (2012) Modeling an autism risk factor in mice leads to permanent immune dysregulation. Proc Natl Acad Sci U S A 109:12776-12781. https://doi.org/10.1073/pnas.1202556109

10. Gee S, Chandiramani M, Seow J, et al (2021) The legacy of maternal SARS-CoV-2 infection on the immunology of the neonate. Nat Immunol. https://doi.org/10.1038/s41590-021-01049-2

11. Faria SS, Fernandes PC, Silva MJB, et al (2016) The neutrophil-to-lymphocyte ratio: a narrative review. Ecancermedicalscience 10:702. https://doi.org/10.3332/ecancer.2016.702

12. Kim S, Eliot M, Koestler DC, et al (2018) Association of Neutrophil-to-Lymphocyte Ratio With Mortality and Cardiovascular Disease in the Jackson Heart Study and Modification by the Duffy Antigen Variant. JAMA Cardiol 3:455-462. https://doi.org/10.1001/jamacardio.2018.1042 
13. Song M, Graubard BI, Rabkin CS, Engels EA (2021) Neutrophil-to-lymphocyte ratio and mortality in the United States general population. Sci Rep 11:464. https://doi.org/10.1038/s41598-020-79431-7

14. Bulut NS, Yorguner N, Çarkaxhiu Bulut G (2021) The severity of inflammation in major neuropsychiatric disorders: comparison of neutrophil-lymphocyte and platelet-lymphocyte ratios between schizophrenia, bipolar mania, bipolar depression, major depressive disorder, and obsessive compulsive disorder. Nord J Psychiatry 1-9. https://doi.org/10.1080/08039488.2021.1919201

15. Tural Hesapcioglu S, Kasak M, Citak Kurt AN, Ceylan MF (2017) High monocyte level and low lymphocyte to monocyte ratio in autism spectrum disorders. Int J Dev Disabil 65:73-81. https://doi.org/10.1080/20473869.2017.1371369

16. Topal Z, Tufan AE, Karadag M, et al (2021) Evaluation of peripheral inflammatory markers, serum B12, folate, ferritin levels and clinical correlations in children with autism spectrum disorder (ASD) and attention deficit hyperactivity disorder (ADHD). Nord J Psychiatry 1-8. https://doi.org/10.1080/08039488.2021.1946712

17. Lord C, Rutter M, Le Couteur A (1994) Autism Diagnostic Interview-Revised: A revised version of a diagnostic interview for caregivers of individuals with possible pervasive developmental disorders. $J$ Autism Dev Disord 24:659-685. https://doi.org/10.1007/BF02172145

18. Lord C, Risi S, Lambrecht L, et al (2000) [No title found]. J Autism Dev Disord 30:205-223. https://doi.org/10.1023/A:1005592401947

19. Forget P, Khalifa C, Defour J-P, et al (2017) What is the normal value of the neutrophil-to-lymphocyte ratio? BMC Res Notes 10:12. https://doi.org/10.1186/s13104-016-2335-5

20. Ellul P, Rosenzwajg M, Peyre H, et al (2021) Regulatory T lymphocytes/Th17 lymphocytes imbalance in autism spectrum disorders: evidence from a meta-analysis. Mol Autism 12:68. https://doi.org/10.1186/s13229-021-00472-4

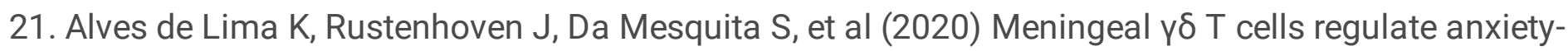
like behavior via IL-17a signaling in neurons. Nat Immunol 21:1421-1429. https://doi.org/10.1038/s41590-020-0776-4

22. Ribeiro M, Brigas HC, Temido-Ferreira M, et al (2019) Meningeal $\gamma \delta T$ cell-derived IL-17 controls synaptic plasticity and short-term memory. Sci Immunol 4:. https://doi.org/10.1126/sciimmunol.aay5199

23. Ito M, Komai K, Mise-Omata S, et al (2019) Brain regulatory T cells suppress astrogliosis and potentiate neurological recovery. Nature 565:246-250. https://doi.org/10.1038/s41586-018-0824-5

24. Xu Z, Zhang X, Chang H, et al (2021) Rescue of maternal immune activation-induced behavioral abnormalities in adult mouse offspring by pathogen-activated maternal Treg cells. Nat Neurosci 24:818-830. https://doi.org/10.1038/s41593-021-00837-1

25. Patel S, Masi A, Dale RC, et al (2017) Social impairments in autism spectrum disorder are related to maternal immune history profile. Mol Psychiatry. https://doi.org/10.1038/mp.2017.201

26. Shimizu Y, Tsukada T, Sakata-Haga H, et al (2021) Exposure to Maternal Immune Activation Causes Congenital Unfolded Protein Response Defects and Increases the Susceptibility to Postnatal 
Inflammatory Stimulation in Offspring. J Inflamm Res 14:355-365.

https://doi.org/10.2147/JIR.S294238

27. Pitoiset F, Cassard L, El Soufi K, et al (2018) Deep phenotyping of immune cell populations by optimized and standardized flow cytometry analyses. Cytom Part J Int Soc Anal Cytol 93:793-802. https://doi.org/10.1002/cyto.a.23570

28. Collier SA, Rasmussen SA, Feldkamp ML, et al (2009) Prevalence of self-reported infection during pregnancy among control mothers in the National Birth Defects Prevention Study. Birt Defects Res A Clin Mol Teratol 85:193-201. https://doi.org/10.1002/bdra.20540

29. Fest J, Ruiter TR, Groot Koerkamp B, et al (2019) The neutrophil-to-lymphocyte ratio is associated with mortality in the general population: The Rotterdam Study. Eur J Epidemiol 34:463-470. https://doi.org/10.1007/s10654-018-0472-y

\section{Supplementary Files}

This is a list of supplementary files associated with this preprint. Click to download.

- 220828suppdata1.docx 